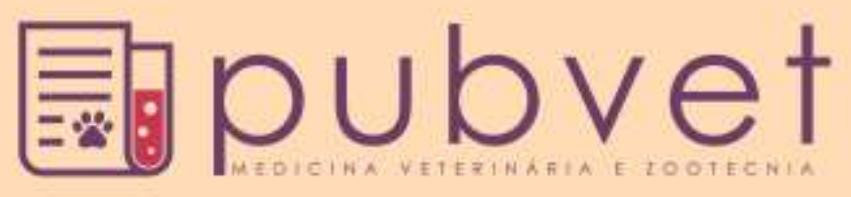

HTTP://DX.DOI.ORG/10.22256/PUBVET.V11N3.262-266

\title{
Anatomia do cone medular do cachorro do mato (Cerdocyon thous) aplicada à via epidural de administração de fármacos
}

\author{
Artur da Nóbrega Carreiro ${ }^{1 *}$, Brunna Muniz Rodrigues Falcão², Fabiana Cristina da \\ Silva Morais ${ }^{3}$, Ana Yasha Ferreira de La Salles ${ }^{1}$, José Rômulo Soares dos Santos ${ }^{4}$, Danilo \\ José Ayres de Menezes ${ }^{5}$, Gildenor Xavier Medeiros ${ }^{5}$ \\ ${ }^{1}$ Aluno de Mestrado do Programa de Pós-graduação em Medicina Veterinária, Universidade Federal de Campina Grande, Unidade \\ Acadêmica de Medicina Veterinária, Patos, Paraíba, Brasil.E-mail: arturpets@gmail.com; anayasha@hotmail.com \\ ${ }^{2}$ Aluna de Graduação em Medicina Veterinária, Universidade Federal de Campina Grande, Unidade Acadêmica de Medicina Veterinária, \\ Patos, Paraíba, Brasil. E-mail: brunnamrfalcao@hotmail.com \\ ${ }^{3}$ Médico Veterinário graduada pela Universidade Federal de Campina Grande, Unidade Acadêmica de Medicina Veterinária, Patos, Paraíba, \\ Brasil. E-mail: fabianamorais@yahoo.com \\ ${ }^{4}$ Técnico do Laboratório de Pesquisas Morfológicas, Universidade Federal de Campina Grande, Unidade Acadêmica de Medicina \\ Veterinária, Patos, Paraíba, Brasil. E-mail: jromulosmedvet@hotmail.com \\ ${ }^{5}$ Professor da Unidade Acadêmica de Medicina Veterinária, Universidade Federal de Campina Grande, Patos, Paraíba, Brasil. Email: \\ mdanayres@gmail.com; gildenorxavier@gmail.com; \\ *Autor de correspondência
}

RESUMO. O acesso à via epidural é de grande importância para diversos procedimentos realizados tanto na clínica como na cirurgia veterinárias. A necessidade de se conhecer a respeito da anatomia e topografia desta região é imprescindível para que o médico veterinário possa obter sucesso em tais técnicas. Com o intuito de determinar a melhor localização para realização da técnica de acesso à via epidural, quer para anestesia epidural, exames de mielografia, coleta de líquor, entre outros, o presente trabalho teve como objetivo descrever a anatomia do cone medular de cachorro do mato (Cerdocyon thous), particularmente sua esqueletopia. Para a realização do presente trabalho seis animais adultos, de ambos os sexos, foram dissecados, retirando-se a musculatura da coluna lombar e sacral para identificação das vértebras, as quais foram seccionadas em seus arcos vertebrais para exposição da medula espinhal. Foi identificado o cone medular, medido com auxílio de paquímetro e identificada a sua esqueletopia. Foi observado, em todos os animais estudados, a presença de sete vértebras lombares (L). O cone medular mostrou-se com a base situada em $\mathrm{L}_{5}\left(50 \%\right.$ dos animais), ou entre $\mathrm{L}_{5}$ e $\mathrm{L}_{6}(50 \%)$ e o ápice em $\mathrm{L}_{6}$ (66.7\%), entre $\mathrm{L}_{6}$ e $\mathrm{L}_{7}(16.7 \%)$ ou em $\mathrm{L}_{7}(16.7 \%)$. O comprimento médio do cone medular observado foi de $16.62+7.33 \mathrm{~mm}$. Como o ápice do cone medular não ultrapassa o espaço lombossacro. Concluímos que neste espaço é possível a realização do acesso ao espaço epidural sem riscos de lesionar a medula espinhal do animal.

Palavras-chave: Anestesia, canídeo, medula espinal, morfometria

\section{Anatomy of the medular cone of crab-eating fox (Cerdocyon thous) applied epidural route of administration of drugs}

ABSTRACT. In order to determine the best location for performing the technique of epidural access either to epidural anesthesia, myelography scans, spinal tap, among others, this study aimed to describe the anatomy of the conus of the crab eating fox (Cerdocyon thous), particularly its esqueletopia. For the realization of this work six adult animals of both sexes were dissected, withdrawing from the muscles of the lumbar and sacral vertebrae column to identify, removing the muscles of the lumbar and sacral vertebrae column to identify which were sectioned in their vertebral arches to expose the spinal cord. Medullary cone was identified, measured with a pachymeter and identified its esqueletopia. In all 
animals studied, the presence of seven lumbar vertebrae (L) were observed. Showed the conus with the base in L5 (50\% of animals), or between L5 and L6 (50\%) in the apex L6 (66.7\%) between L6 and L7 (16.7\%) or in L7 (16.7\%). The average length of the medullary cone observed was $16.62+7.33 \mathrm{~mm}$. As the apex of the conus does not exceed the lumbosacral space. We concluded that this space is possible the realization of access to the epidural space without risk of injuring the spinal cord of the animal.

Keywords: Canid, spinal anesthesia, Spinal cord, morphometry

\title{
Anatomía del cono medular del perro de monte (Cerdocyon thous) aplicada a la vía epidural de administración de fármacos
}

\begin{abstract}
RESUMEN. El acceso a la vía epidural es de gran importancia para muchos procedimientos realizados tanto en cirugía clínica y veterinaria. La necesidad de conocer la anatomía y la topografía de esta región es vital para que el veterinario puede tener éxito en tales técnicas. Con el intuito de determinar la mejor localización para la realización de la técnica de acceso a la vía epidural, requiere para anestesia epidural, pruebas de mielografía, colecta de líquido, entre otros, este estudio tuvo como objetivo describir la anatomía del cono medular (extremo distal de la médula espinal) del perro de monte (Cerdocyon thous), en particular su esqueletopia. Para llevar a cabo este trabajo fueron disecados seis animales adultos de ambos sexos, retirándose la musculatura de columna lumbar y sacra para identificación de las vértebras, las cuales fueron seccionadas en sus arcos vertebrales para exposición de la médula espinal. Fue identificado el cono medular, medido con auxilio del paquímetro e identificada su esqueletopatia. Se observó en todos los animales estudiados, la presencia de siete vértebras lumbares $(\mathrm{L})$. El cono medular se mostró con la base situada en L5 (50\% de los animales), o entre L5 y L6 (50\%) y el vértice en L6 (66,7\%) entre L6 y L7 (16,7\%), o L7 ( 16,7\%). La largura media del cono medular observado fue $16,62+7.33 \mathrm{~mm}$. Como el ápice do cono medular no ultrapasa el espacio lumbosacro. Concluimos que en este espacio es posible llevar a cabo el acceso al espacio epidural sin riesgo de lesionar la médula espinal del animal.
\end{abstract}

Palabras clave: Anestesia, canino, medula espinal, morfometria

\section{Introdução}

Popularmente conhecido por raposa ou cachorro do mato (Rocha et al., 2008), a Cerdocyon thous é o canídeo silvestre mais comum do continente sul-americano, apresentando coloração grisalha, com alguns pelos negros, que podem variar individualmente (Pinheiro et al., 2011, Ramos Júnior et al., 2003). Por serem vítimas constantes de acidentes automobilísticos, caçadores, ou mesmo de armadilhas utilizadas para proteger a pecuária e agricultura, eventualmente são encaminhados a clinicas e hospitais veterinários. Da mesma forma, alguns espécimes são mantidos em zoológicos e/ou em centros de preservação e podem vir a necessitar de cuidados clínicos, o que exige do Médico Veterinário o conhecimento sobre os aspectos anatomofisiológico desses animais.

$\mathrm{O}$ acesso à via epidural é importante para a realização de anestesia epidural lombossacra e anestesia peridural contínua, sendo técnicas lógicas para anestesia e analgesia cirúrgica utilizadas em cães que são considerados de risco para anestesia inalatória ou intravenosa (Tranquilli et al., 2013).

Para a realização da anestesia epidural é imprescindível o conhecimento da localização exata do cone medular, podendo trazer complicações quando não executada de forma adequada, com riscos de lesões de medula espinhal. Em pequenos animais o acesso para anestesia epidural é feito preferencialmente no espaço lombossacro e em grandes animais (bovinos e equinos) no espaço intercoccígeo (Massone, 1999). Entretanto, segundo Silva et al. (2009), em gatos domésticos o cone medular apresenta-se com topografia bastante variada e diferente da apresentada pelos cães, o que deve ser considerado ao se eleger o local mais adequado para o acesso epidural.

Com o intuito de determinar a melhor localização para o acesso ao espaço epidural para realização de anestesia epidural, bem como exames de mielografia e coleta de líquor, o 
presente trabalho teve como objetivo descrever a topografia do cone medular de cachorro do mato (C. thous).

\section{Material e Métodos}

Para a realização do presente trabalho foram utilizados seis cachorros do mato da espécie Cerdocyon thous, de ambos os sexos, adultas, cedidas pelo Laboratório de Virologia ao Laboratório de Anatomia Veterinária, ambos da Universidade Federal de Campina Grande, Campus de Patos - Paraíba - Brasil, (licença de captura IBAMA $\left.\mathrm{N}^{\circ} 332 / 2001,2002\right)$ e identificados por meio de medidas craniométricas.

Os animais encontravam-se fixados e conservados em solução aquosa de formol a $10 \%$. Para exposição de toda a coluna vertebral, rebatendo-se a pele e retirou-se a musculatura da região dorsal, identificando-se particularmente as vértebras lombares e sacrais. Posteriormente, com auxílio de um paquímetro digital com precisão de 0,01 mm (Paquímetro digital eletrônico, DIGIMESS) foi localizado e medido o comprimento longitudinal (crânio-caudal) e transversal (látero-lateral) do espaço lombossacro. Essas medições foram realizadas com os animais fixados em posição anatômica e em decúbito lateral.

Para estabelecer o final da medula espinhal e medir o comprimento do cone medular foi aberto todo o canal vertebral lombossacro, seccionandose lateralmente os arcos vertebrais. Em seguida a duramáter foi seccionada para visualização do cone medular e observação da relação topográfica deste com as vértebras.

Para medir o comprimento do cone medular foi utilizado novamente o paquímetro digital, medindo-se desde a base até o ápice do cone.

Os dados coletados foram analisados pelo programa Assistat - assistência estatística (2007) e estão expressos em média e desvio padrão.

\section{Resultados}

Os resultados observados demonstraram variações na topografia do cone medular, bem como, no tamanho do cone e do espaço lombossacro em C. thous.

Para melhor referência da esqueletopia do cone medular, foram identificadas e contadas as vértebras lombares e sacrais e foi observado que os animais apresentaram sete vértebras lombares e três sacrais.
Conforme observado na Tabela 1, a base do cone medular está situada entre a $5^{\mathrm{a}}$ e $6^{\mathrm{a}}$ vértebras lombares em três animais $(50 \%)$ e no nível da $5^{\text {a }}$ vértebra lombar nos demais (50\%). Na maioria dos animais estudados o ápice estava situado no nível da $6^{\mathrm{a}}$ vértebra lombar (66.7\%) (Figura 1), entretanto em um animal (16.7\%) estava entre a $6^{\mathbf{a}}$ e $7^{\mathrm{a}}$ vértebra lombar e em outro $(16.7 \%)$ na $7^{\mathrm{a}}$ vértebra lombar.

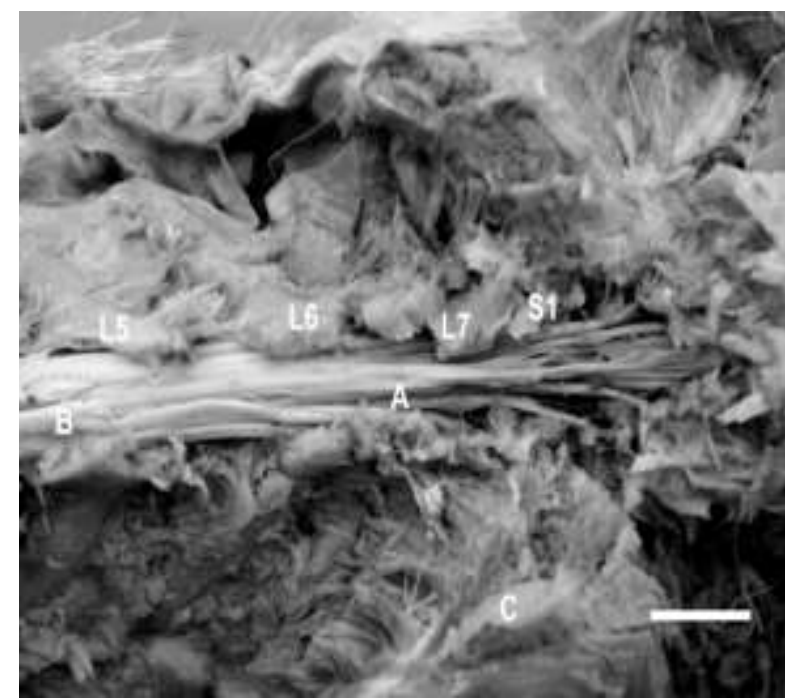

Figura 1. Fotografia do cone medular de cachorro do mato (Cerdocyon thous). Evidencia-se a $5^{\mathrm{a}}$ (L5), $6^{\mathrm{a}}$ (L6), $7^{\mathrm{a}}$ (L7) vértebras lombares e $1^{\mathrm{a}}(\mathrm{S} 1)$ vértebra sacral, bem como a crista isquiática esquerda (C). Nota-se a base (B) do cone medular em L5 e o ápice $(\mathrm{A})$ entre L6 e L7 $($ Barra $=1 \mathrm{~cm})$.

Tabela 1. Frequência da topografia do cone medular em cachorro do mato (Cerdocyon thous)

\begin{tabular}{lcccc}
\hline Topografia & \multicolumn{3}{c}{ Base } \\
\cline { 2 - 5 } & \multicolumn{2}{c}{$\begin{array}{c}\text { Número } \\
\%\end{array}$} & $\begin{array}{c}\text { Frequência, } \\
\%\end{array}$ & $\begin{array}{c}\text { Número } \\
\%\end{array}$ \\
\hline L5 & 3 & 50 & - & - \\
Entre L5 e L6 & 3 & 50 & - & - \\
L6 & - & - & 4 & 66,7 \\
Entre L6 e L7 & - & - & 1 & 16,7 \\
L7 & - & - & 1 & 16,7 \\
\hline Total & 6 & 100 & 6 & 100 \\
\hline
\end{tabular}

O espaço lombossacro (Figura 2) variou tanto em comprimento como em largura (Tabela 2). O comprimento do cone medular variou de $9.79 \mathrm{~mm}$ a $29.50 \mathrm{~mm}$, com média de $16.62+7.33 \mathrm{~mm}$ (Tabela 2). Longitudinalmente o diâmetro variou de $4.64 \mathrm{~mm}$ a $6.98 \mathrm{~mm}$, com a média de $5.65 \pm$ $0.88 \mathrm{~mm}$. Transversalmente variou de $5.90 \mathrm{~mm}$ a $9.41 \mathrm{~mm}$, com a média de $7.18 \mathrm{~mm}$.

\section{Discussão}

Os cachorros do mato (C. thous) observados apresentaram sete vértebras lombares; diferente 
do descrito por Pinheiro et al. (2011) em relato de um caso da mesma espécie, os quais verificaram nove vértebras lombares.

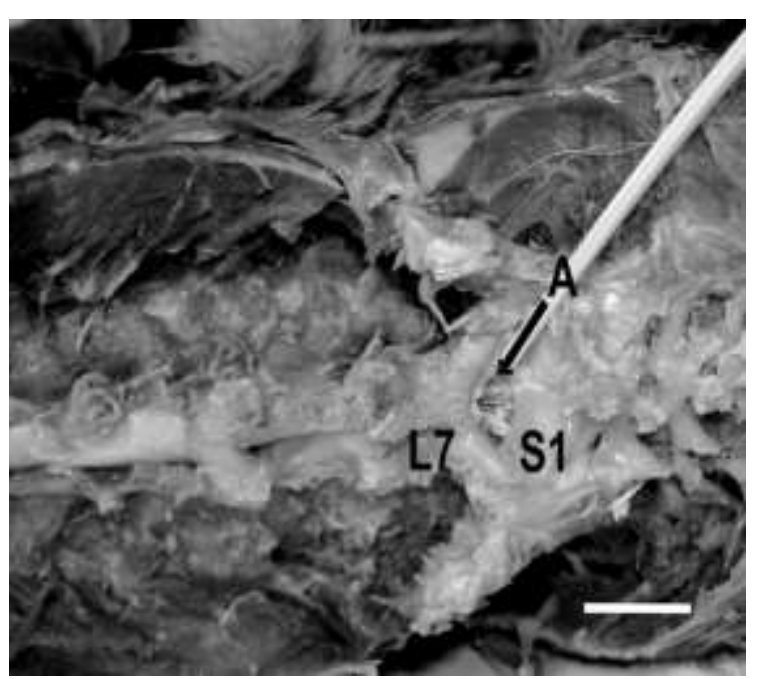

Figura 2. Vista dorsal do espaço lombossacro (A), da $7^{\mathrm{a}}$ vértebra lombar (L7) e da $1^{\text {a }}$ vértebra sacral $(\mathrm{S} 1)$ de cachorro do mato (Cerdocyon thous) $($ Barra $=0,5 \mathrm{~cm})$.

Tabela 2. Comprimento do cone medular e diâmetro do espaço lombossacro em cachorro do mato (Cerdocyon thous)

\begin{tabular}{lccc}
\hline Animal & $\begin{array}{c}\text { Comprimento } \\
\text { do cone, mm }\end{array}$ & $\begin{array}{c}\text { Diâmetro do espaço lombo-sacro } \\
\text { Longitudinal, } \\
\mathrm{mm}\end{array}$ & Transversal mm \\
\hline $\mathrm{N}^{\mathrm{o}} 1$ & 11,19 & 5,29 & 6,59 \\
$\mathrm{~N}^{\circ} 2$ & 9,79 & 5,97 & 7,34 \\
$\mathrm{~N}^{\mathrm{o}} 3$ & 12,33 & 4,64 & 6,67 \\
$\mathrm{~N}^{\circ} 4$ & 17,59 & 6,68 & 9,41 \\
$\mathrm{~N}^{\circ} 5$ & 19,30 & 5,40 & 5,90 \\
$\mathrm{~N}^{\circ} 6$ & 29,50 & - & - \\
\hline Média & 16,62 & 5,65 & 7,18 \\
$\mathrm{SD}$ & 7,33 & 0,88 & 1,35 \\
\hline
\end{tabular}

A topografia do ápice do cone medular, que corresponde ao final da medula espinhal, variou entre a $6^{\mathrm{a}}$ e $7^{\mathrm{a}}$ vértebras lombares, aproximando-se do descrito no lobo guará (Chrysocium brachiurus), onde o final da medula variou da $5^{\mathrm{a}}$ a $6^{a}$ vértebra lombar (Machado et al., 2002). Apesar da grande variação da esqueletopia do cone medular relatada pelos autores no cão (Canis familiaris), observa-se semelhança desses animais com os cachorros do mato estudados. Assim, a topografia do cone medular do cão pode termina entre a $4^{\mathrm{a}}$ e $5^{\mathrm{a}}$ vértebra lombar (Bruni and Zimmer, 1947) podendo alcançar a $7^{\text {a }}$ vértebra lombar, como relatado em $32.5 \%$ de 40 cães estudados por Santiago (1974). Segundo Dyce et al. (2004) e Sisson et al. (1986) estas grandes variações na topografia do cone medular são comuns nos caninos em função da grande quantidade de raças.

Em comparação ao relatado por Pinheiro et al. (2011), os quais descreveram o cone medular com a base na $6^{\mathrm{a}}$ vértebra lombar e o ápice ao nível da $3^{\text {a }}$ vértebra sacral em um exemplar do cachorro do mato, no presente estudo o ápice do cone medular não ultrapassou o espaço lombossacro alcançando, no máximo, a última vértebra lombar, com a base entre a $5^{\mathrm{a}}$ ou $6^{\mathrm{a}}$ vértebra lombar.

O comprimento médio do cone medular foi, de todas as medidas, a que ocorreu maior variação, com média de $16.62 \pm 7.33 \mathrm{~mm}$, a qual foi inferior ao observado no lobo guará ( $C$. brachiurus) que apresentou comprimento médio de $2.5 \mathrm{~cm}$ (Machado et al., 2002). Diferença também foi observada entre os animais deste estudo e o cachorro do mato descrito por Pinheiro et al. (2011), o qual afirmou que o cone medular do $C$. thous media $10 \mathrm{~cm}$.

Em relação ao diâmetro do espaço lombossacro, foi verificado uma média longitudinal de $5.65 \pm 0.88 \mathrm{~mm}$ e transversal de $7.18 \pm 1.35 \mathrm{~mm}$. Essas medidas se aproximam das encontradas por Hopkins (1935) em caninos com peso médio $4.98 \mathrm{~kg}$, em que as medidas foram de $4.0 \mathrm{~mm}$ longitudinal e $7.0 \mathrm{~mm}$ transversal. Vale ressaltar que essas medidas foram aferidas com o animal em posição anatômica, pois o diâmetro longitudinal pode aumentar em animais vivos com a flexão sacro-pélvica, favorecendo a punção lombossacra, especialmente para anestesia epidural. Lumb and Jones (1984) e De Lahunta et al. (2014) indicam o espaço lombossacro como o de eleição para anestesia epidural em cães, gatos, suínos e ovelhas.

As diferenças encontradas entre o cachorro do mato deste estudo e os descritos por Pinheiro et al. (2011), apesar de identificado pelo autor como sendo da mesma espécie, por se tratar de relato de caso com um animal, pode ter ocorrido erro do autor na identificação da espécie ou mesmo variações anatômicas no indivíduo estudado, já que as diferenças de posição e tamanho do cone, bem como do número de vértebras lombares são marcantes.

A topografia do cone medular do cachorro do mato foi estudada e identificada com o intuito de colaborar com a aplicação do conhecimento na prática de anestesia epidural na espécie, tendo em vista que, segundo Hopkins (1935) a eficiência, segurança e a rapidez para a utilização prática dos 
métodos de anestesia epidural dependem diretamente do conhecimento da anatomia da região envolvida. Bone and Peck (1956) mencionam uma lista de indicações para anestesia epidural em cães: cesariana, ovariohisterectomia, castração, redução de prolapso retal, caudectomia, mastectomia, excisão de tumores nos membros pélvicos, redução de fraturas e relaxamento do abdômen para facilitar a palpação.

\section{Conclusão}

Concluiu-se que, apesar da variação do comprimento do cone medular de cachorro do mato (Cerdocyon thous), o seu ápice não ultrapassa a sétima vértebra lombar, ou seja, o espaço lombossacro, permitindo o acesso a via epidural neste espaço sem riscos de lesionar a medula espinhal do animal.

\section{Referências Bibliográficas}

Bone, J. K. \& Peck, J. G. 1956. Epidural anesthesia in dogs. Journal of the American Veterinary Medical Association, 128, 236-238.

Bruni, A. C. \& Zimmer, U. 1947. Anatomia degli animali domestici. Francesco Vallardi, Milano.

De Lahunta, A., Glass, E. N. \& Kent, M. 2014. Veterinary neuroanatomy and clinical neurology. W.B. Saunders, Philadelphia.

Dyce, K. M., Wensing, C. J. G. \& Sack, W. O. 2004. Tratado de anatomia veterinária. Elsevier Brasil, São Paulo.

Hopkins, G. S. 1935. The correlation of anatomy and epidural anesthesia in domestic mammals, Cornell.

Lumb, W. V. \& Jones, E. W. 1984. Veterinary anesthesia. Lea \& Febiger, Philadelphia.

Machado, G. V., Fonseca, C. C., Neves, M. T. D., Paula, T. A. R. \& Benjamin, L. A. 2002. Topografia do cone medular no lobo-guará (Chrysocyon brachyurus llliger, 1815). Revista Brasileira de Ciência Veterinária, 9, 107-109.

Massone, F. 1999. Anestesiologia veterinária. Guanabara Koogan, Rio de Janeiro.
Pinheiro, L. L., Lima, A. R., Souza, A. C. B., Pereira, L. C. \& Branco, É. R. 2011. Topografia do cone medular do cachorro-domato (Cerdocyon thous Linnaeus, 1766): relato de caso. Biotemas, 24, 129-133.

Ramos Júnior, V. A., Pessutti, C. \& Chieregatto, C. A. S. 2003. Guia de identificação dos canídeos silvestres brasileiros. Biotemas, 24, 129-133.

Rocha, V. J., Aguiar, L. M., Silva-Pereira, J. E., Moro-Rios, R. F. \& Passos, F. C. 2008. Feeding habits of the crab-eating fox, Cerdocyon thous (Carnivora: Canidae), in a mosaic area with native and exotic vegetation in Southern Brazil. Revista Brasileira de Zoologia, 25, 594-600.

Santiago, W. 1974. Esqueletopia do cone medular em Canis familiaris. Arquivo da Universidade Federal Rural do Rio de Janeiro, 4, 67-69.

Silva, P. H. C., Silva, R. M. \& Lima, E. M. M. 2009. Topografia do cone medular em gatos sem raça definida. Arquivo Brasileiro de Medicina Veterinária e Zootecnia, 61, 10621066.

Sisson, S., Grossman, J. D. \& Getty, R. 1986. Anatomia dos animais domésticos. Interamericana, Rio de Janeiro.

Tranquilli, W. J., Thurmon, J. C. \& Grimm, K. A. 2013. Lumb and Jones' veterinary anesthesia and analgesia. John Wiley \& Sons, Iowa, USA.

\section{Article History:}

Received 29 November 2016

Accepted 4 December 2016

Available on line 10 February 2017

License information: This is an open-access article distributed under the terms of the Creative Commons Attribution License 4.0, which permits unrestricted use, distribution, and reproduction in any medium, provided the original work is properly cited. 